\title{
Inhibition of Phosphodiesterase-4 (PDE4) activity triggers luminal apoptosis and AKT dephosphorylation in a 3-D colonic-crypt model
}

Toshiyuki Tsunoda ${ }^{1,2 \dagger}$, Takeharu Ota ${ }^{1,2,3 \dagger}$, Takahiro Fujimoto ${ }^{1,2}$, Keiko Doi ${ }^{1,2}$, Yoko Tanaka ${ }^{1,2}$, Yasuhiro Yoshida ${ }^{1}$, Masahiro Ogawa ${ }^{2}$, Hiroshi Matsuzaki ${ }^{1}$, Masato Hamabashiri ${ }^{1}$, Darren R Tyson ${ }^{4}$, Masahide Kuroki ${ }^{2}$,

Shingo Miyamoto ${ }^{3}$ and Senji Shirasawa $a^{1,2^{*}}$

\begin{abstract}
Background: We previously established a three-dimensional (3-D) colonic crypt model using HKe3 cells which are human colorectal cancer (CRC) HCT116 cells with a disruption in oncogenic KRAS, and revealed the crucial roles of oncogenic KRAS both in inhibition of apoptosis and in disruption of cell polarity; however, the molecular mechanism of KRAS-induced these 3-D specific biological changes remains to be elucidated.

Results: Among the genes that were upregulated by oncogenic KRAS in this model, we focused on the phosphodiesterase $4 B$ (PDEAB) of which expression levels were found to be higher in clinical tumor samples from CRC patients in comparison to those from healthy control in the public datasets of gene expression analysis. PDE4B2 was specifically overexpressed among other PDE4 isoforms, and re-expression of oncogenic KRAS in HKe3 cells resulted in PDE4B overexpression. Furthermore, the inhibition of PDE4 catalytic activity using rolipram reverted the disorganization of HCT116 cells into the normal physiologic state of the epithelial cell polarity by inducing the apical assembly of ZO-1 (a tight junction marker) and E-cadherin (an adherens junction marker) and by increasing the activity of caspase-3 (an apoptosis marker) in luminal cavities. Notably, rolipram reduced the AKT phosphorylation, which is known to be associated with the disruption of luminal cavity formation and CRC development. Similar results were also obtained using PDE4B2-shRNAs. In addition, increased expression of PDE4B mRNA was found to be correlated with relapsed CRC in a public datasets of gene expression analysis.

Conclusions: These results collectively suggested that PDE4B is upregulated by oncogenic KRAS, and also that the inhibition of PDE4 catalytic activity can induce both epithelial cell polarity and luminal apoptosis in CRC, thus highlighting the utility of our 3-D culture (3 DC) model for the KRAS-induced development of CRC in 3-D microenvironment. Indeed, using this model, we found that PDE4B is a promising candidate for a therapeutic target as well as prognostic molecular marker in CRC. Further elucidation of the signaling network of PDE4B2 in 3 DC would provide a better understanding of CRC in vivo.
\end{abstract}

Keywords: PDE4B, Three-dimensional culture, Colorectal cancer, Kras, AKT

\footnotetext{
* Correspondence: sshirasa@fukuoka-.ac.jp

${ }^{\dagger}$ Equal contributors

'Department of Cell Biology, Faculty of Medicine, Fukuoka University,

Fukuoka 814-0180, Japan

${ }^{2}$ Central Research Institute for Advanced Molecular Medicine, Fukuoka

University, Fukuoka 814-0180, Japan

Full list of author information is available at the end of the article
} 


\section{Background}

Both cell-cell and cell-extracellular matrix interactions are critically involved in developmental programs and provide three-dimensional (3-D) architectures in vivo [1], and deregulation of these interactions is frequently observed in cancer [2]. Human cancers are derived from epithelial tissues characterized by specific cellular architectures including epithelial cell-cell junctions, which allow the separation of apical and basolateral membranes. This apical-basal cell polarity is crucial in normal cell functions, and loss of cell polarity is a critical step in tumorigenesis [3].

We recently demonstrated that $\mathrm{HKe} 3$ cell line, which is a human colorectal cancer (CRC) HCT116 cell line disrupted at oncogenic KRAS [4], in 3-D Matrigel culture (3 DC) manifests an organized structure resembling a colonic crypt [5]. In this model, oncogenic KRAS was found to be involved in the inhibition of luminal apoptosis, impairment of epithelial cell polarity and downregulation of DNA repair genes including TP53 and $B R C A 2$ in a 3-D specific manner [5], suggesting that this model could mimic the in vivo growth of the colonic epithelium and would be useful for determining the critical genes involved in CRC development through oncogenic KRAS-mediated signals in vivo.

We previously identified phosphodiesterase 4B (PDE4B) as one of the differentially expressed between HCT116 cells and HKe3 cells in this model [5]. PDE4 cyclic AMP (cAMP)-specific phosphodiesterase family members are hydrolytic enzymes responsible for the degradation of the second messenger cAMP in many cell types, and the family consists of four genes (PDE4A, PDE4B, PDE4C and PDE4D) encoding multiple isoforms [6-8]. These isoforms can have unique functional roles by their targeting to specific signaling complexes where they underpin the compartmentalization of cAMP signaling [9]. Notably, particular PDE4 isoforms are subject to different regulatory influences, such as phosphorylation [10], ubiquitination [11] and activity changes induced by interacting proteins [12]. For example, the interaction of the disrupted in schizophrenia 1 (DISC1) with PDE4B1 or PDE4B3 and mutations in DISC1 associates with schizophrenia [13].

PDE4 is ubiquitously expressed in inflammatory cells and PDE4 inhibitors have a therapeutic potential for inflammatory diseases, including asthma, chronic obstructive pulmonary diseases, inflammatory bowel disease and psoriasis [14]. A recent study suggests that overexpression of PDE4 enzymes is critical for the MAPK activation by oncogenic-RAS in melanoma cells [15], indicating the novel strategy targeting PDE4 activity in melanoma cells with oncogenic KRAS. However, the precise mechanism of PDE4B in 3-D microenvironment of CRC with oncogenic KRAS has not been addressed so far.
In this study, we used a selective inhibitor of all PDE4 sub-families, rolipram $[9,14,16]$ or PDE4B2-shRNAs, and found that rolipram and PDE4B2-shRNAs revert the disorganization of CRC into the normal physiologic state of the epithelial cell in 3 DC.

\section{Results}

Commonly upregulated genes between HCT116 cells in 3 DC and clinical CRC samples

To explore the genes regulated by oncogenic KRAS in $3 \mathrm{DC}$, we analyzed the microarray expression data for HCT116 and HKe3 cells grown in 3 DC on day 6 [5] using the GenePattern software package [17]. We selected the 387 genes with a score of more than 10 as the differentially expressed genes between HCT116 and HKe3 cells grown in 3 DC (Figure 1A, Additional file: 1 Table S1). Then, out of the 387 genes selected, we further selected 25 genes with a score of more than three as the differentially expressed genes between 12 human colorectal specimens and 10 colonic mucosa specimens in a public datasets [18] (Figure 1B, C). Among the 25 genes, we focused on PDE4B, which was suggested to be associated with luminal cavity formation [19].

\section{PDE4B is upregulated by oncogenic KRAS in 3 DC}

In our microarray expression analyses, the relative expression level of $P D E 4 B$ in 3 DC was increased in HCT116 cells compared with that of HKe3 cells, whereas the relative expression levels of the other PDE4 family members, including PDE4A, PDE $4 C$ and PDE4D, were decreased in HCT116 cells in comparison to those in HKe3 cells (Figure 2A).

To confirm these results, quantitative RT-PCR (qRTPCR) for PDE4 family was performed on HKe3 cells and HCT116 cells in 3 DC. In HKe3 cells, PDE4B and $P D E 4 D$ were abundantly expressed than PDE4A or $P D E 4 C$. The expression level of PDE4B in HCT116 cells was significantly increased by 3.4 -fold in comparison to that in HKe3 cells ( $\left.{ }^{* *} P=0.0008\right)$, whereas the expression level of PDE4D in HCT116 cells was significantly decreased by 183 -fold in comparison to that in HKe3 cells (Figure $2 \mathrm{~B} ;{ }^{*} P=0.01$ ), indicating that the oncogenic KRAS will regulate PDE4B and PDE4D positively and negatively, respectively.

To elucidate which isoform of PDE4B or PDE4D is exactly expressed, qRT-PCR was performed on HKe3 cells and HCT116 cells in 3 DC. Among PDE4B and PDE4D isoforms, the expression level of PDE4B2 in HCT116 cells was only increased in comparison to that in HKe3 cells (Figure $2 C$; $" P<0.01$ ). On the other hand, the expression levels of PDE4D1 and PDE4D2 in HCT116 cells were significantly decreased in comparison to these in HKe3 cells (Figure $2 \mathrm{C} ;{ }^{*} \mathrm{P}<0.01$ ), indicating that the oncogenic 


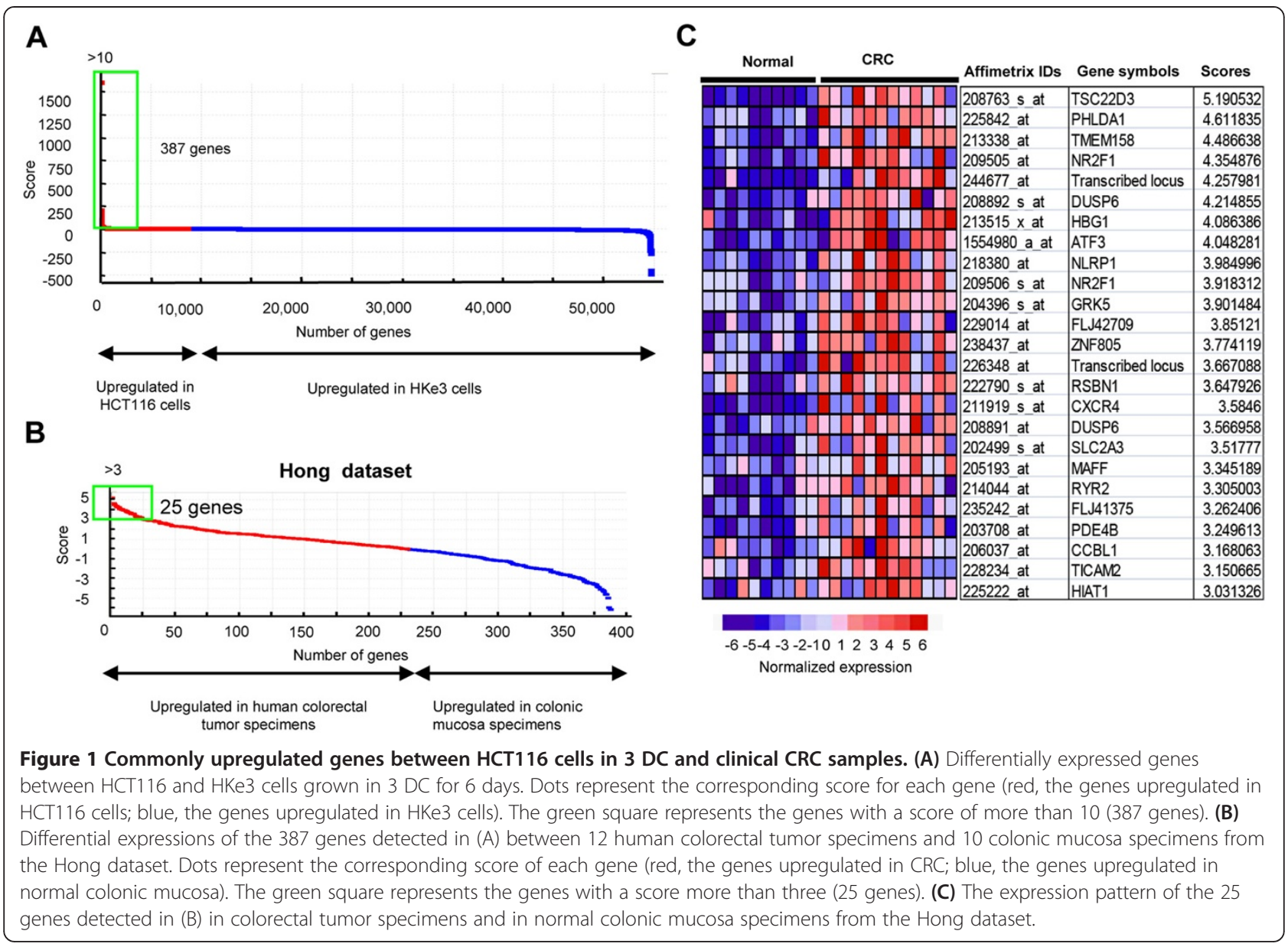

KRAS will positively regulate PDE4B2, and negatively regulate PDE4D1 and PDE4D2.

To confirm the oncogenic KRAS-mediated upregulation of PDE4B, qRT-PCR was performed on HKe3 cells, HCT116 cells and e3-MKRas \#14 cells re-expressing oncogenic KRAS. In two-dimensional culture (2 DC), the expression levels of PDE4B in HCT116 cells and e3MKRas\#14 cells were increased by 3.6 - and 4.0-fold $\left({ }^{*} P<0.05\right)$, respectively, in comparison to that in the HKe3 cells (Figure 2D). In 3 DC, the expression levels of PDE4B in HCT116 cells and e3-MKRas\#14 cells were increased by 7.3- and 11.2-fold ( $P<0.05)$, respectively, in comparison to that in the HKe3 cells (Figure 2D). These results suggest that oncogenic KRAS in two dimensional (2-D) and 3-D cultures upregulates the mRNA expression of PDE4B. Furthermore, the expression levels of PDE4B in HCT116 and e3-MKRas\#14 cells in 3 DC were increased by 2.0 - and 2.8 -fold $(* * P<0.01)$, respectively, in comparison to those in $2 \mathrm{DC}$, whereas the expression level of $P D E 4 B$ in the HKe3 cells in 3 DC was not significantly different in comparison to that in $2 \mathrm{DC}$ (Figure 2D). These results together suggest that PDE4B, especially PDE4B2, plays particular roles in the 3-D microenvironment.
Formation of luminal cavities and tight junctions after treatment with PDE4 inhibitor in HCT116 cells

To address the roles of PDE4B in cell polarity, ZO-1 (a tight junction marker) and E-cadherin (an adherens junction marker) were immunostained in HCT116 cells grown in $3 \mathrm{DC}$ treated with rolipram (PDE4 inhibitor) or DMSO alone. The ZO-1 and E-cadherin assembly at the apical surface of acini was more clearly observed in HCT116 cells in 3 DC treated with rolipram in comparison to that in DMSO alone (Figure 3A). These results indicated that rolipram induces the formation of the junctional complexes essential for the maintenance of the physiologic epithelial cell polarity [20] in HCT116 cells grown in $3 \mathrm{DC}$.

To confirm these results, the quantitative assays were performed. The ratio of 3-D structures with luminal cavities in HCT116 cells treated with rolipram was increased by 2.46 -fold in comparison to those treated with DMSO alone (Figure 3B left panel; ${ }^{*} P=0.00009$ ), thus suggesting that rolipram induces the formation of the luminal cavity. The ratio of the 3-D structures with the concentrated signals for ZO-1 in the apical region of the HCT116 cells treated with rolipram increased by 7.67 -fold in 


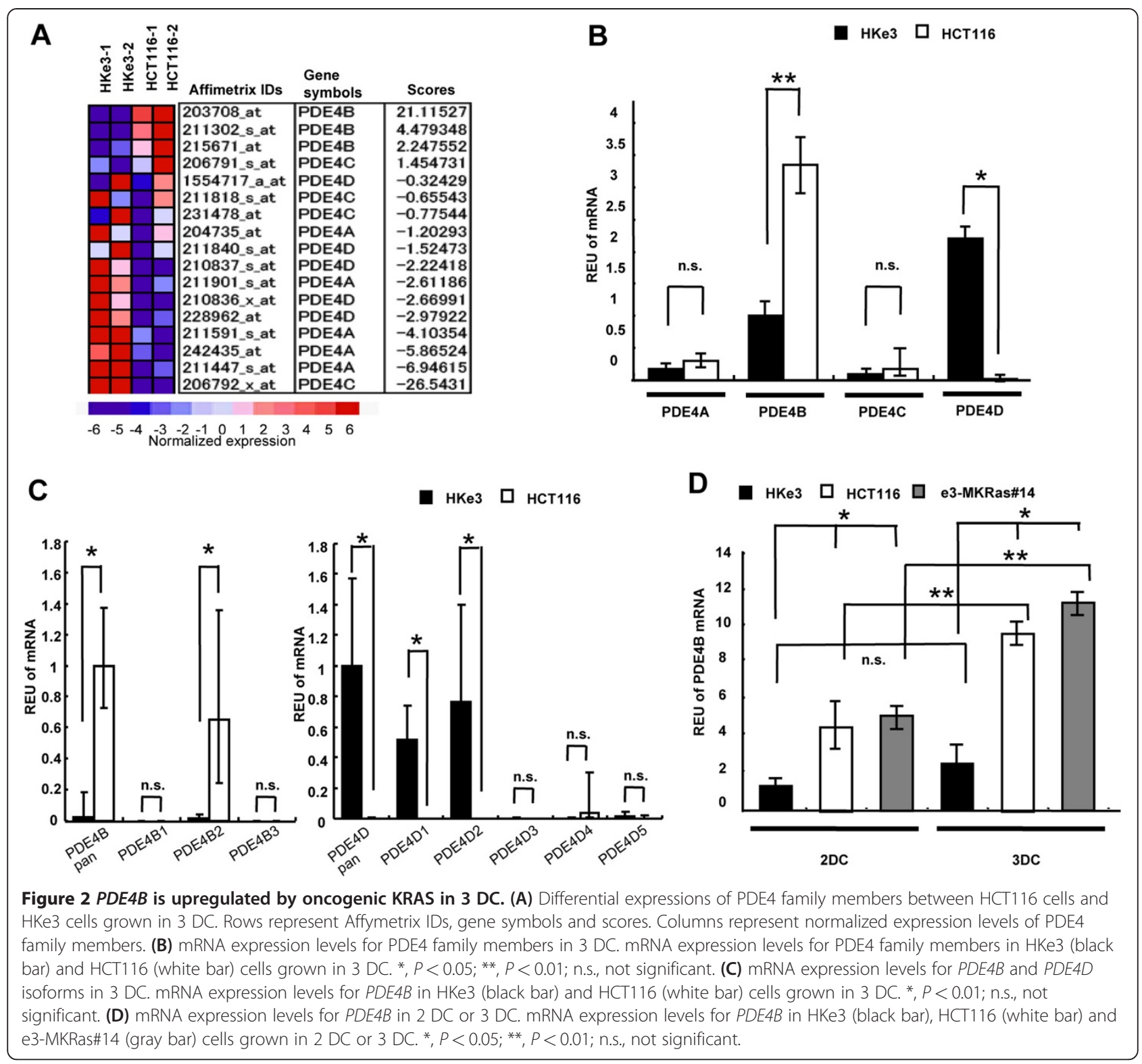

comparison to those treated with DMSO alone (Figure 3B right panel; $" P=0.00002$ ), thus suggesting that rolipram induces the formation of tight junctions. As the expression levels of ZO-1 were not significantly different between HCT116 cells treated with DMSO alone or rolipram (Figure 3C; $P=0.09$ ), ZO-1 was properly translocated to the tight junction at the apical surface of the 3-D structures by reduction of the activity of PDE4B.

\section{Induction of luminal apoptosis by rolipram in HCT116 cells grown in 3 DC}

To address the roles of PDE4B in the process of colonic crypt organization, we compared the differences in cell proliferation and luminal apoptosis between $\mathrm{HKe} 3$ and HCT116 cells grown in 3 DC treated with rolipram or
DMSO alone. The proliferation rate detected by Ki-67 staining in $3 \mathrm{DC}$ on day 3 was not different between HKe3 cells treated with DMSO alone and those treated with rolipram (Figure 4A). Similar result was obtained in HCT116 cells treated with DMSO alone and those treated with rolipram (Figure 4A). These results indicated that rolipram does not affect cell proliferation in $\mathrm{HKe} 3$ or HCT116 cells grown in 3 DC on day 3.

To address whether rolipram influences luminal apoptosis in $3 \mathrm{DC}$, we evaluated the apoptotic activity in HKe3 and HCT116 cells grown in 3 DC for 6 days with DMSO alone or rolipram by detecting the signals for cleaved caspase-3 in each 3-D structure using confocal microscopy. Without the treatment of rolipram, the ratio of the 3-D structures containing apoptotic cells in 


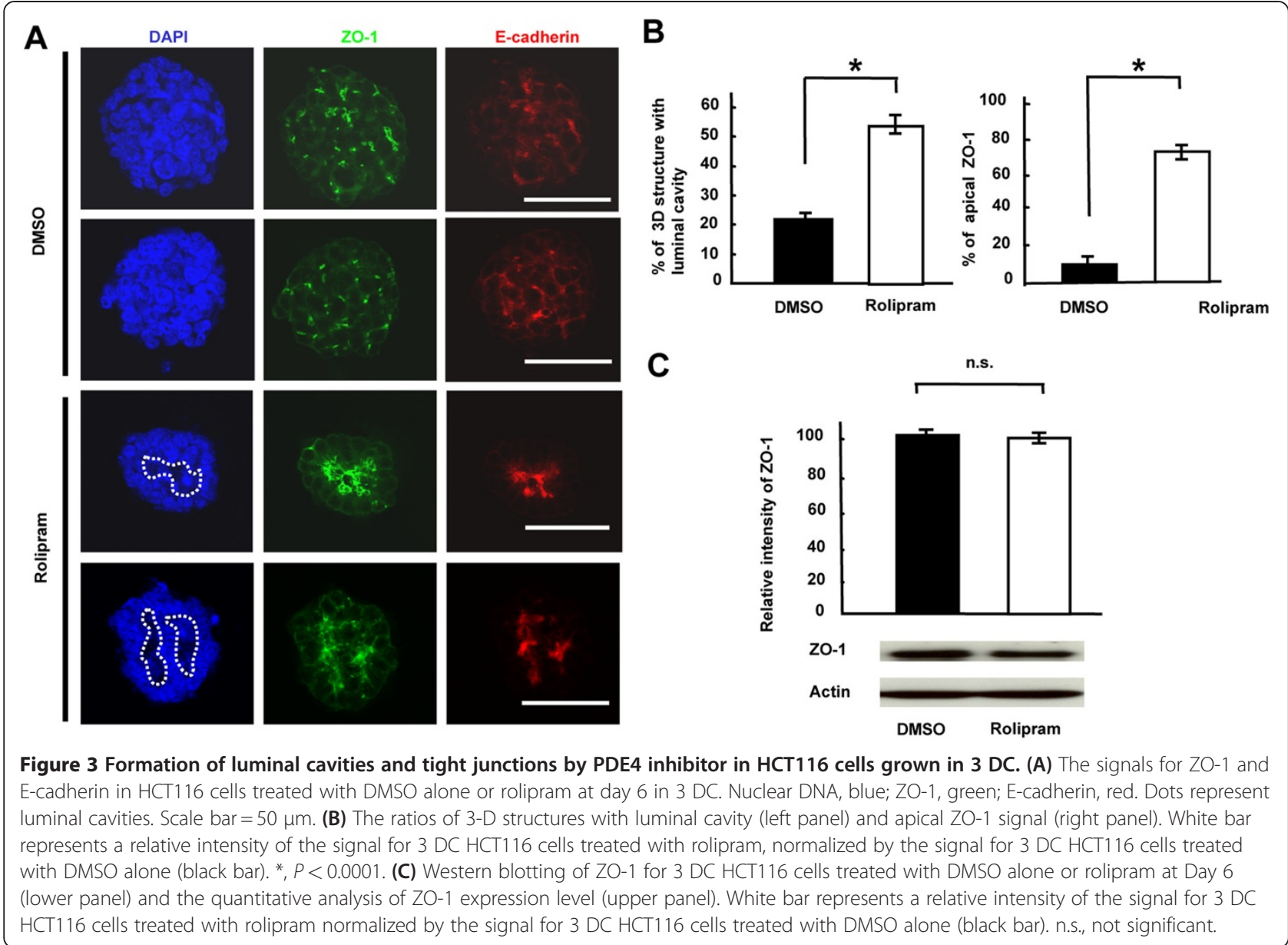

HCT116 cells was decreased by 3.77 -fold in comparison to that for HKe3 cells (Figure $4 \mathrm{~B} ;{ }^{*} P=0.00002$ ), which observation was concordant with the previous report [5]. The ratios of the 3-D structures containing apoptotic cells in $\mathrm{HKe} 3$ cells treated with DMSO alone or rolipram were not different (Figure $4 \mathrm{~B} ;{ }^{*} P=0.25$ ), suggesting that rolipram does not further affect luminal apoptosis in the cells already having developed luminal cavity. On the other hand, the ratio of the 3-D structures containing apoptotic cells in the HCT116 cells treated with rolipram was found to be increased by 2.21 -fold in comparison to those treated with DMSO alone (Figure 4B; $\left.{ }^{*} P=0.0004\right)$, suggesting that a physiological apoptosis can be restored by the inhibition of PDE4 catalytic activity.

\section{Induction of luminal apoptosis by PDE4B2-shRNAs in} HCT116 cells grown in 3 DC

To asses the direct role of PDE4B2 gene, we established stable HCT116 transfectants expressing control shRNA or PDE4B2-shRNAs through the use of lentivirus. A qRT-PCR analysis showed that the expression level of PDE4B2 mRNA in the HCT116 cells expressing the
PDE4B2-shRNA \#2 and \#5 were significantly decreased compared with those in HCT116 cells transfected with the control shRNA (Figure $5 \mathrm{~A} ;{ }^{*} P<0.005$ ). To examine whether PDE4B2-shRNAs affect cytoplasmic cAMP level, we performed the enzyme-linked immunosorbent assay for cAMP in these HCT116 cells transfected with control shRNA or PDE4B2-shRNAs in 2-D or 3-D culture. The expected cAMP levels were detected in all cell lines, however, the reduced expression of PDE4B2 did not affect cytoplasmic cAMP levels as reported before [21] in 2-D or 3-D culture (Figure 5B). To address whether PDE4B2-shRNAs influence luminal apoptosis in $3 \mathrm{DC}$, we evaluated the apoptotic activity in these cells grown in $3 \mathrm{DC}$ for 6 days by detecting the signals for cleaved caspase- 3 using confocal microscopy. In HCT116 cells with control shRNA, the ratio of the 3-D structures containing apoptotic cells was decreased by 3.30 - and 3.13-fold in comparison to that for HCT116 cells with PDE4B2-shRNA \#2 and \#5, respectively (Figure $5 C ; * P<0.005$ ). This observation was similar with the result obtained in Figure $3 \mathrm{~B}$, suggesting that a physiological apoptosis can be restored by the direct inhibition of expression level of PDE4B2 mRNA. 


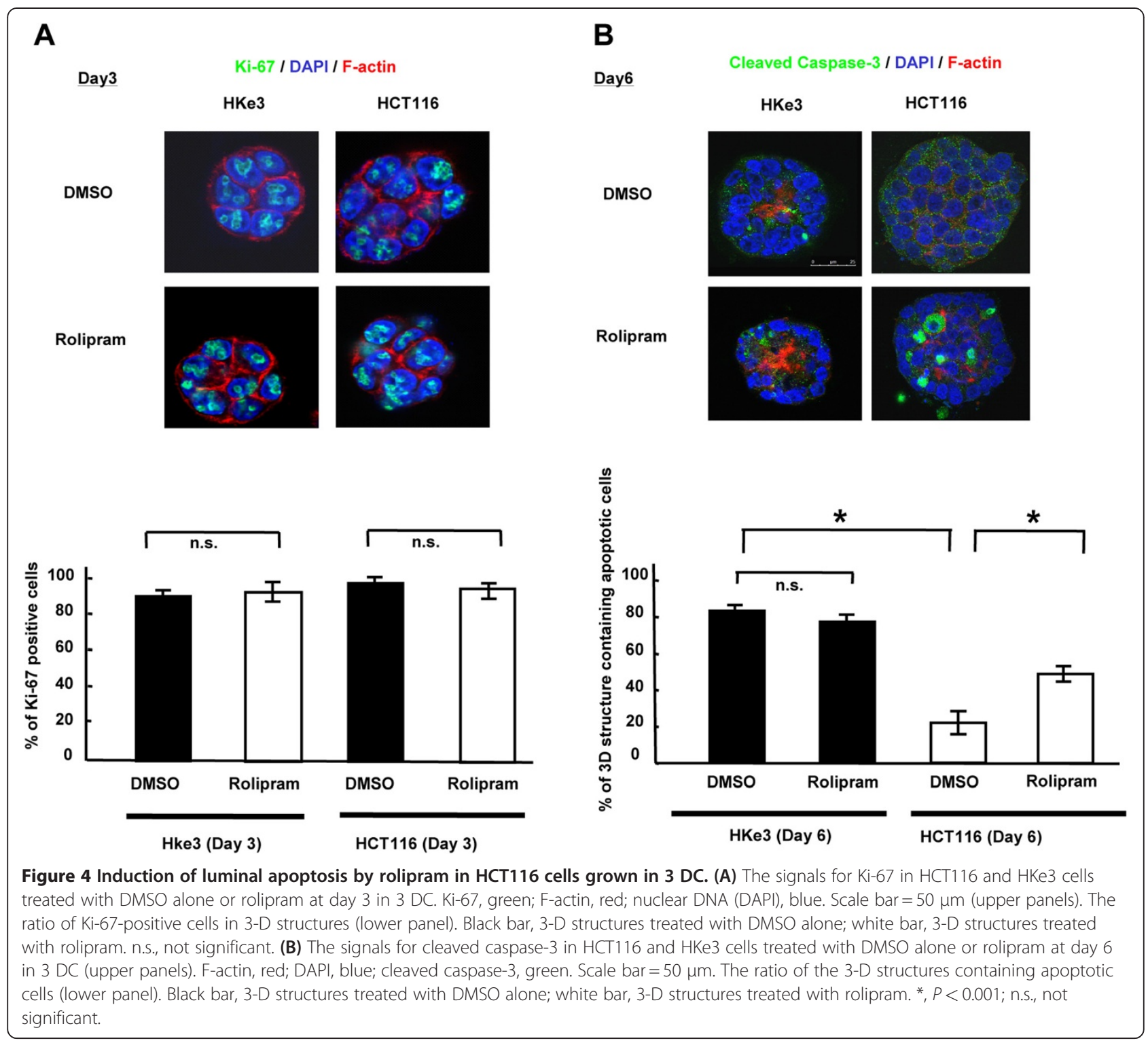

\section{3-D-specific reduction in AKT phosphorylation by rolipram or PDE4B2-shRNAs in HCT116 cells}

$\mathrm{AKT}$ is reported to play a major role in regulating acinar structure [22], and PDE4B is suggested to be a selective modulator for AKT phosphorylation at Ser473 [23]. Therefore, we examined the effect of rolipram on phosphorylation of AKT at S473, which is associated with enhanced AKT activity, in CRC cells in 2 DC and 3 DC. While the level of the AKT phosphorylation in HCT116 cells in 2 DC was not affected by rolipram, its phosphorylation was decreased by 2.70 -fold in the HCT116 cells grown in $3 \mathrm{DC}$ in response to rolipram treatment (Figure 6A and $6 \mathrm{~B} ;{ }^{*} P=0.003$ ). Additionally, we also evaluated the phosphorylation of AKT at Thr308, however, any significant difference was not observed (Figure 5A). Similarly, the level of the AKT phosphorylation at Ser473 in HCT116 cells in 2 DC was not affected by PDE4B2 -shRNAs. On the other hand, the levels of AKT phosphorylation at Ser473 were decreased by 1.51 - and 1.67fold in the HCT116 cells with PDE4B2-shRNA \#2 and \#5 grown in $3 \mathrm{DC}$, respectively (Figure $6 C ; * P<0.05$ ). These results suggested that the oncogenic KRAS will disrupt the acinar structure, in part, through the regulation of AKT phosphorylation by increasing the activity of PDE4B in the 3-D microenvironment.

\section{Correlation between increased PDE4B expression and disease relapse in CRC patients}

Recent studies indicated that 3 DC mimics the early step of metastasis process [24] and the differentially expressed genes between organized and unorganized multicellular structures grown in $3 \mathrm{DC}$ share similarities with the 

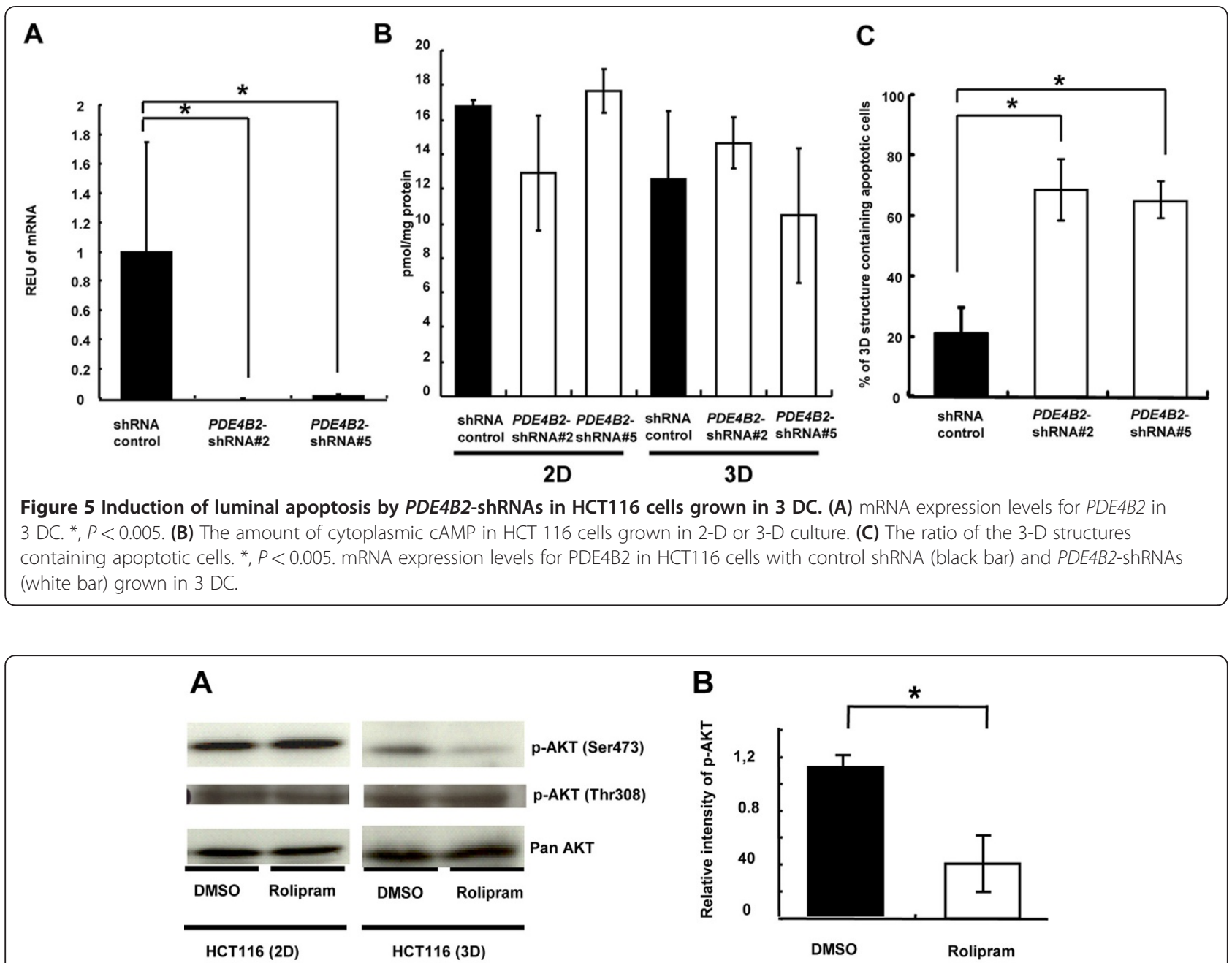

C

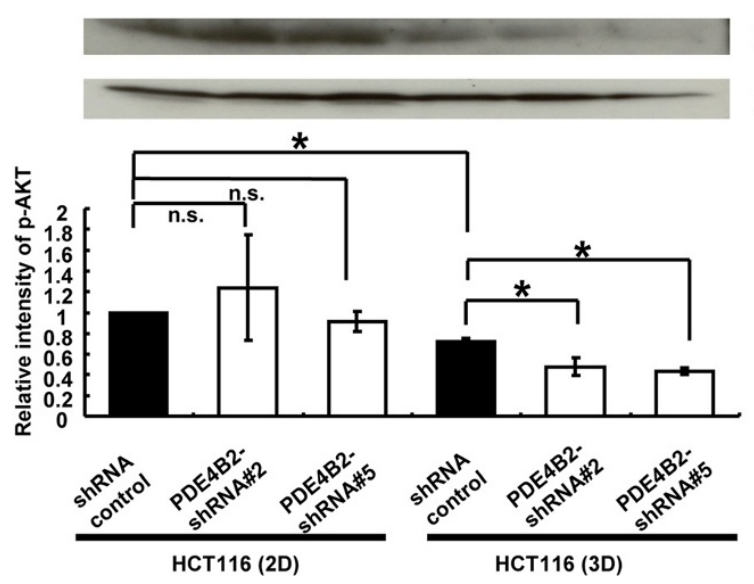

Figure 6 Reduction of AKT phosphorylation by rolipram in HCT116 cells grown in 3 DC. (A) Western blotting of AKT phosphorylation (pAKT) at Ser473 and Thr308, and pan-AKT for HCT116 cells treated with DMSO alone or rolipram at day 6 in 2-D or 3-D culture. (B) The quantitative analyses of the level of p-AKT (Ser473) in HCT116 cells treated with DMSO alone or rolipram at day 6 in 3 DC. White bar represents the relative intensity of the signal for HCT116 cells treated with rolipram, normalized by the signal for HCT116 cells treated with DMSO alone (black bar). ${ }^{*}, P<0.01$. (C) Upper panel shows western blotting of p-AKT (Ser473) and pan-AKT for HCT116 cells stably transfected with control shRNA or PDE4B2-shRNAs at day 6 in 2-D or 3-D culture. Lower panel shows the quantitative analyses of the level of p-AKT in HCT116 cells stably transfected with control shRNA or PDE4B2-shRNAs at day 6 in 3 DC. White bar represents the relative intensity of the signal for HCT116 cells stably transfected with PDE4B2-shRNAs, normalized by the signal for HCT116 cells stably transfected with control shRNA (black bar). ${ }^{*} P<0.05$. 
differentially expressed genes between good- and poorprognosis tumors [25-27]. To examine the correlation between PDE4B expression and the prognosis of clinical colorectal tumors, we analyzed 18 genes out of 25 KRASupregulated genes (Figure 1B, C) in a public datasets of the microarray-based gene expression analyses of human colorectal tumor specimens from 6 CRC patients in the relapsed group and those from 10 patients in nonrelapsed group in a public datasets [28], revealing that expression of $P D E 4 B$ mRNA was significantly upregulated in the relapsed group compared with the non-relapsed group (Figure 6), thus suggesting the critical involvement of PDE4B in tumor progression and poor prognosis.

\section{Discussion}

In polarized cells, the inactivation of AKT is thought to be important for the lumen formation with apoptosis in 3 DC $[22,29,30]$. The findings of AKT dephosphorylation by PDE4 inhibitor, rolipram or PDE4B2-shRNAs in HCT116 cells in 3 DC, but not in HCT116 cells in 2 DC (Figure 4 and 5), suggesting the 3-D specific action of PDE4B2 for cancer cells with oncogenic KRAS.

In clinical samples, increased expression level of $P D E 4 B$ mRNA was correlated with disease relapse in CRC patients (Figure 7). Furthermore, among 25 KRASupregulated genes (Figure $1 \mathrm{~B}, \mathrm{C}$ ), the predictive power of PDE4B expression for poor prognosis is stronger than that of CXCR4 which is reported to be a prognostic factor for poor disease outcome [31,32]. PDE4B is also reported to be predictive of the resistance to EGFR tyrosine kinase inhibitors in human lung tumors with KRAS mutation [33]. These reports indicate that PDE4B is a promising candidate for a prognostic marker in CRC.

Several studies have shown the effectiveness of PDE4 inhibitors. A recent study shows that resveratrol binds with PDE4 and ameliorates aging-related metabolic phenotypes [34], indicating the positive health benefits through the use of PDE4 inhibitors. In cancer, several studies shows the anti-tumor effects of rolipram in combination with forskolin were observed in different types of cancer, including hematological $[23,35,36]$, brain $[37,38]$ and skin cancers [15]. In CRC, Murata et al., reported that the constitutive activation of PDE4 was detected in a colon cancer cell line DLD-1, and rolipram suppressed cellular motility [39]. McEwan et al., reported that the 10 $\mu \mathrm{M}$ (the same dose used in our study) of rolipram in combination with an adenylate cyclase activator (forskolin) induced apoptosis in colon cancer cell line KM12C [40]. These reports suggested that the apoptotic reaction was induced through the increased stability of cAMP by PDE4 inhibitor or the increased activity of cAMP by forskolin [40]. Notably, our study demonstrated that luminal apoptosis was effectively induced by rolipram alone (Figure 4) or PDE4B2-shRNAs (Figure 5) without affecting cytoplasmic cAMP level (Figure 5B) in HCT116 cells in 3 DC, suggesting that the effect of PDE4 inhibitor is enhanced by 3-D microenvironment and independent of cAMP level. Indeed, ablation of PDE4B, but not PDE4A or PDE4D, causes a major decrease in TNF production and global change in cAMP could not be detected in $\mathrm{PDE}_{4 \mathrm{~B}}{ }^{-/-}$macrophage, together, suggesting PDE4B2 may have specific role among PDE4B isoforms. For example, PDE4B2 is highly expressed in immune cell and associated with diffuse large B cell lymphoma [35]. In melanoma, the increased PDE4B2 expression was observed compared with that in melanocyte and PDE4B2 expression is necessary to transform melanocyte with oncogenic NRAS [15], implicating the effective strategy for cancer treatment by targeting the PDE4B2.

\section{Conclusions}

This is a first report about the correlation between the inhibition of PDE4 catalytic activity and luminal cavity formation in CRC cells with oncogenic KRAS grown in 3 DC. Oncogenic KRAS upregulates the PDE4B expression and the inhibition of PDE4 catalytic activity by rolipram or the PDE4B2-shRNAs triggers the induction of polarity, apoptosis and AKT dephosphorylation in HCT116 cells grown in $3 \mathrm{DC}$, thus suggesting the increased PDE4B2 activity will be crucial in the inhibition of luminal apoptosis in colorectal epithelium in 3-D microenvironment. Further elucidation of the precise molecular mechanisms of function of PDE4B would provide a better understanding of the development and progression of CRC.

\section{Methods}

\section{Antibodies and reagents}

The anti-Ki-67 (SP6) was obtained from Thermo Scientific (Rockford, IL). The anti-ZO-1 (1/ZO-1) and anti-Ecadherin (36/E-cadherin) were from BD Biosciences (San Jose, CA). Anti-cleaved caspase-3 (5A1), antiphosphorylated AKT (p-AKT; D9E) and the anti-AKT (pan; C67E7) were from Cell Signaling Technology (Beverly, MA). The anti-actin (A2066), the PDE4 inhibitor, rolipram and 4',6-diamidino-2-phenylindole (DAPI) were from Sigma-Aldrich (St Louis, MO).

\section{Cell culture}

Human CRC HCT116 cells were obtained from the American Type Culture Collection (Frederick, MD). Twodimensional (2-D) culture (2 DC) of HCT116 cells, HKe3 cells and e3-MKRas\#14 cells (HKe3-derived stable transfectants expressing oncogenic KRAS) was done as described previously $[4,5,41]$. The $3 \mathrm{DC}$ was performed using Matrigel, a reconstituted basement membrane (Growth Factor Reduced Matrigel; BD bioscience), as described previously [5,42]. All cell lines used were 


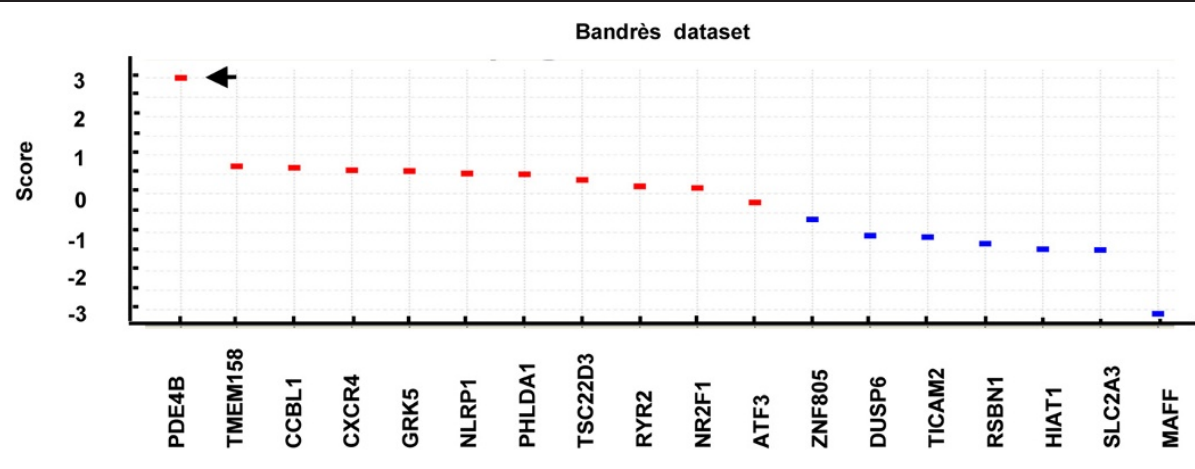

Relapse

$(-)$

$(+)$

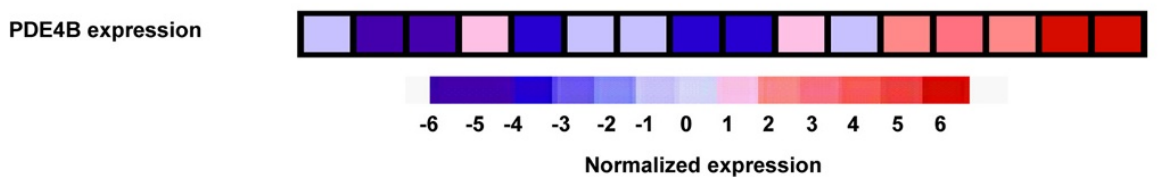

Figure 7 Correlation between increased PDE4B expression and disease relapse in CRC patients. Differential expressions of 18 genes between colorectal tumor specimens from six CRC patients in the relapsed group and those from 10 patients in non-relapsed group in Bandrès dataset. Dots in upper panel represent the score (red, upregulated genes in relapsed group; blue, upregulated genes in non-relapsed group). Arrowhead represents PDE4B. Columns in lower panel represent normalized expression levels of PDE4B in 16 Dukes' B colorectal tumor specimens.

confirmed to be Mycoplasma-free, as determined using the MycoAlert system (Lonza, Verviers, Belgium). Cell morphology was regularly checked to ensure the absence of cross-contamination of cell lines.

\section{Microarray expression analysis}

Total RNA from cells in 3 DC was extracted as described previously [5,43]. Human Genome U133 Plus 2.0 Array 6800 GeneChips (Affymetrix, Santa Clara, CA) containing 54,675 oligonucleotides were processed with the use of robust multi-chip averaging [44]. The differentially expressed genes between two classes were ranked according to signal-to-noise metric with the GenePattern software package $[17,45]$. The statistical significance of the differentially expressed genes was determined by the comparative marker selection module in Gene-Pattern [17].

\section{Dataset source}

The Hong dataset, consisting of the microarray profiles of human colorectal tumor specimens from 12 CRC patients and colonic mucosa specimens from 10 healthy control subjects [18], was obtained from the Gene Expression Omnibus (GEO; Series GSE4107). The Bandrès dataset, consisting of human Dukes' B colorectal tumors from 16 CRC patients with associated clinical data [28], was also obtained from the GEO (Series GSE2630). GEO series records were imported by the import module in the GenePattern software package [17]. The platform used for Hong dataset was Human Genome U133 Plus
2.0 Array 6800 GeneChips (Affymetrix), which was identical to our previous study [5]. As the platform used for Bandrès dataset was Human $19 \mathrm{~K}$ Oligo array slides (Center for Applied Genomics, University of Medicine of New Jersey), 25 probe set IDs in Human Genome U133 Plus 2.0 Array 6800 GeneChips (Affymetrix) were converted to gene symbols and 18 genes were identified out of the 25 probe set IDs.

\section{Quantitative RT-PCR}

Quantitative RT-PCR was performed using ABI PRISM 7900HT (Applied Biosystems, Carlsbad, CA) as described previously $[5,43]$. The PCR primer sequences used for PDE4 isoforms and the accession numbers for the genes are listed in Additional file: 2 Table S2. The PCR primers used for PDE4A (pan), PDE4C (pan) and PDE4D (pan) were designed to amplify a 3' fragment found in all PDE4 sub-families as reported before [7]. The PCR primers used for PDE4B (pan) were designed to amplify a fragment characteristic of human PDE4B1 and PDE4B2 as reported before [7]. The PCR primers used for PDE4B and PDE4D isoforms were designed as described previously [46]. The relative expression unit (REU) values were determined by the setting REU of HKe3 in 2 DC as 1.0.

\section{Immunofluorescence labeling and confocal microscopy} The immunofluorescence experiments were performed as described previously $[5,47]$. For the examination of 
3-D structures, TCS-SP5 Laser Scanning confocal microscopy (Leica, Wetzlar, Germany) was used.

\section{Quantification of apical ZO-1 signals and luminal cavities in 3-D structures}

The cells were stained with an anti-ZO-1, anti-Ecadherin antibody and DAPI on day 6 , and the ratio of 3-D structures with ZO-1 signals and the luminal cavities in the apical region were counted as described previously [5]. A total of 60 of the 3-D structures from three different wells were counted.

\section{Quantification of proliferative cells grown in 3 DC}

The cells were stained with the anti-Ki-67 antibody, DAPI and phalloidin on day 3 . The ratio of Ki-67-positive cells in the total cells contacting matrigel in the cross-section of 3-D structures at maximum diameter was calculated as described previously [5]. The number of 3-D structures evaluated for each condition was 20 .

\section{Quantification of apoptosis in 3-D structures}

The cells were stained by anti-cleaved caspase- 3 antibody, DAPI and phalloidin on day 6. The cleaved caspase-3-positive cells in 3-D structures were counted in the serial cross-sections of the 3-D structure ranged from 60 to $130 \mu \mathrm{m}$ in the maximum diameter. The 3-D structures containing more than two positive cells with luminal cavity or actin assembly at the apical surface of acini were defined as the 3-D structure containing apoptotic cells. A total of 60 of the 3-D structures from three different wells were counted. The 3-D structures of HCT116 and HKe3 cells were analyzed in three independent experiments, and the average ratio of the 3-D structures containing apoptotic cells was calculated as described previously [5].

\section{Western blotting analysis}

The western blotting analyses were performed as described previously [5]. The actin intensity was used as a control in the western blot analyses for $\mathrm{ZO}-1$, and the relative intensity of the signal (ZO-1/Actin) was normalized to the signal-intensity in HCT116 cells treated with DMSO alone (as 100\%). Pan-AKT intensity was used as a control in the western blotting analyses for $\mathrm{p}-\mathrm{AKT}$, and the relative intensity of the signal (p-AKT/pan-AKT) was normalized to the signal intensity in HCT116 cells treated with DMSO alone as $100 \%$.

\section{Generation of lentivirus vectors expressing PDE4B2- shRNAs}

The short hairpin interfering RNA (shRNA) targeting GFP was used as a control. For PDE4B2 knockdown, shRNAs were designed based on the sequence information from PDE4B2-siRNAs used in the study for diffuse large B-cell lymphoma [35]. The shRNA duplexes used were: PDE4B2-shRNA \#2 top, 5'-CAC CGC CTA AAC AAT ACA AGC ATT TCA AGA GAA TGC TTG TAT TGT TTA GGC-3' and PDE4B2-shRNA \#2 bottom, 5'-AAA AGC CTA AAC AAT ACA AGC ATT CTC TTG AAA TGC TTG TAT TGT TTA GGC-3'; PDE4B2-shRNA \#5 top, 5'CAC CGC ATC TCA CGC TTT GGA GTT TCA AGA GAA CTC CAA AGC GTG AGA TGC-3' and PDE4B2-shRNA \#5 bottom, 5'- AAA AGC ATC TCA CGC TTT GGA GTT CTC TTG AAA CTC CAA AGC GTG AGA TGC-3'. The shRNA expression vectors were constructed as described previously [48]. In brief, The human U6 promoter (Gene bank accession \#M14486 gene sequence 65-329) was inserted into ClaI and SalI sites of the pLenti6/V5-Dest (Invitrogen, Carlsbad, CA, USA), and then U6-term was inserted into the SalI and MluI sites to form pLenti6-U6 + term. The resulting pLenti6-U6+term was then cleaved with $B s m B I$ to form a cloning site for doublestranded synthetic oligonucleotide DNA.

\section{shRNA transfection}

The shRNA expression vectors were transfected into 293FT cells to produce packaged lentivirus. The lentivirus particles were packaged using the ViraPower Lentiviral Expression System (Invitrogen). The HCT116 cells were then infected with lentivirus PDE4B2-shRNAs to obtain stably transfected clones. Blasticidin (Invitrogen) was added to eliminate the cells not expressing PDE4B2shRNAs.

\section{cAMP analysis}

Cytoplasmic protein extraction was performed using NE-PER Nuclear and Cytoplasmic Extraction Reagent (Thermo Scientific) according to the manufacturers instructions. cAMP levels of cytoplasmic extract from 2-D or 3D culture were measured using the direct cAMP ELISA kit (Enzo Life Sciences, Farmingdale, NY, USA) according to the manufacturer's instructions.

\section{Statistical analysis}

The data are presented as the means \pm standard deviation. The statistical analyses were performed using unpaired two-tailed Student's $t$-test. Differences at $P<0.05$ were considered to be statistically significant.

\section{Additional files}

Additional file 1: Additional Table 1. Upregulated genes by oncogenic KRAS in 3 DC (HCT116/HKe3 in 3 DC; Score > 10.00; 387 genes). 
Additional file 2: Additional Table 2. List of the forward and reverse primers used to amplify PDE4 variants and $\beta$-actin.

\section{Abbreviations}

KRAS: v-Ki-ras 2 Kirsten rat sarcoma viral oncogene homolog; AKT: Akt murine thymoma viral oncogene homolog; TP53: Tumor protein 53; BRCA2: Breast cancer gene 2; MAPK: Mitogen-activated protein kinase; ZO-1: Zonula occludens-1; EGFR: Epidermal growth factor receptor.

\section{Competing interests}

The authors declare that they have no competing interests.

\section{Authors' contributions}

TT performed the statistical analysis, coordinated the experiments, and drafted the manuscript. TO carried out the cell and molecular biology experiments and drafted the manuscript. TF, YT, YY, MO and HM performed the cellular assays, molecular studies, western blots and RT-PCR. KD carried out the microarray gene expression analysis and bioinformatics. DRT contributed interpretation and analyzed data. MK and SM participated in study design. SS contributed to data evaluation, interpretation and drafted the manuscript. All authors have read and approved the final manuscript.

\section{Acknowledgements}

We thank Takami Danno and Yumiko Hirose for their technical assistance. This study was supported, in part, by a grant from the Ministry of Education Culture, Sports, Science and Technology of Japan (MEXT), MEXT-Supported Program for the Strategic Research Foundation at Private Universities, 2008-2012 and from the Clinical Research Foundation.

\section{Author details}

'Department of Cell Biology, Faculty of Medicine, Fukuoka University, Fukuoka 814-0180, Japan. ${ }^{2}$ Central Research Institute for Advanced Molecular Medicine, Fukuoka University, Fukuoka 814-0180, Japan. ${ }^{3}$ Department of Obstetrics and Gynecology, Faculty of Medicine, Fukuoka University, Fukuoka 814-0180, Japan. ${ }^{4}$ Department of Cancer Biology, Vanderbilt University, Nashville, TN, USA

Received: 3 November 2011 Accepted: 25 July 2012

Published: 25 July 2012

\section{References}

1. O'Brien LE, Zegers MM, Mostov KE: Opinion: Building epithelial architecture: insights from three-dimensional culture models. Nat Rev Mol Cell Biol 2002, 3:531-537.

2. Griffith $L G$, Swartz MA: Capturing complex 3D tissue physiology in vitro. Nat Rev Mol Cell Biol 2006, 7:211-224.

3. Wodarz A, Nathke I: Cell polarity in development and cancer. Nat Cell Biol 2007, 9:1016-1024.

4. Shirasawa S, Furuse M, Yokoyama N, Sasazuki T: Altered growth of human colon cancer cell lines disrupted at activated Ki-ras. Science 1993, 260:85-88.

5. Tsunoda T, Takashima Y, Fujimoto T, Koyanagi M, Yoshida Y, Doi K, Tanaka $Y$, Kuroki M, Sasazuki T, Shirasawa S: Three-dimensionally specific inhibition of DNA repair-related genes by activated KRAS in colon crypt model. Neoplasia 2010, 12:397-404.

6. Spina D: PDE4 inhibitors: current status. Br J Pharmacol 2008, 155:308-315.

7. Erdogan S, Houslay MD: Challenge of human Jurkat T-cells with the adenylate cyclase activator forskolin elicits major changes in CAMP phosphodiesterase (PDE) expression by up-regulating PDE3 and inducing PDE4D1 and PDE4D2 splice variants as well as down-regulating a novel PDE4A splice variant. Biochem J 1997, 321(Pt 1):165-175.

8. Conti M, Beavo J: Biochemistry and physiology of cyclic nucleotide phosphodiesterases: essential components in cyclic nucleotide signaling. Annu Rev Biochem 2007, 76:481-511.

9. Houslay MD: Underpinning compartmentalised CAMP signalling through targeted cAMP breakdown. Trends Biochem Sci 2009, 35:91-100.

10. Houslay MD, Baillie GS, Maurice DH: CAMP-Specific phosphodiesterase-4 enzymes in the cardiovascular system: a molecular toolbox for generating compartmentalized cAMP signaling. Circ Res 2007, 100:950-966.
11. Li X, Baillie GS, Houslay MD: Mdm2 directs the ubiquitination of betaarrestin-sequestered cAMP phosphodiesterase-4D5. J Biol Chem 2009, 284:16170-16182.

12. Bolger GB, Peden AH, Steele MR, MacKenzie C, McEwan DG, Wallace DA, Huston E, Baillie GS, Houslay MD: Attenuation of the activity of the CAMPspecific phosphodiesterase PDE4A5 by interaction with the immunophilin XAP2. J Biol Chem 2003, 278:33351-33363.

13. Millar JK, Pickard BS, Mackie S, James R, Christie S, Buchanan SR, Malloy MP, Chubb JE, Huston E, Baillie GS, et al: DISC1 and PDE4B are interacting genetic factors in schizophrenia that regulate CAMP signaling. Science 2005, 310:1187-1191.

14. Page CP, Spina D: Phosphodiesterase inhibitors in the treatment of inflammatory diseases. Handb Exp Pharmacol 2011, 204:391-414.

15. Marquette A, Andre J, Bagot M, Bensussan A, Dumaz N: ERK and PDE4 cooperate to induce RAF isoform switching in melanoma. Nat Struct Mol Biol 2011, 18:584-591.

16. Keravis T, Lugnier C: Cyclic nucleotide phosphodiesterases (PDE) and peptide motifs. Curr Pharm Des 2010, 16:1114-1125

17. Reich M, Liefeld T, Gould J, Lerner J, Tamayo P, Mesirov JP: GenePattern 2.0. Nat Genet 2006, 38:500-501.

18. Hong Y, Ho KS, Eu KW, Cheah PY: A susceptibility gene set for early onset colorectal cancer that integrates diverse signaling pathways: implication for tumorigenesis. Clin Cancer Res 2007, 13:1107-1114.

19. Mangoo-Karim R, Uchic M, Lechene C, Grantham JJ: Renal epithelial cyst formation and enlargement in vitro: dependence on CAMP. Proc Nat Acad Sci U S A 1989, 86:6007-6011.

20. Miyoshi J, Takai Y: Molecular perspective on tight-junction assembly and epithelial polarity. Adv Drug Deliv Rev 2005, 57:815-855.

21. Jin SL, Lan L, Zoudilova M, Conti M: Specific role of phosphodiesterase 4B in lipopolysaccharide-induced signaling in mouse macrophages. $\lrcorner$ Immunol 2005, 175:1523-1531.

22. Debnath J, Walker SJ, Brugge JS: Akt activation disrupts mammary acinar architecture and enhances proliferation in an mTOR-dependent manner. J Cell Biol 2003, 163:315-326.

23. Smith PG, Wang F, Wilkinson KN, Savage KJ, Klein U, Neuberg DS, Bollag G, Shipp MA, Aquiar RC: The phosphodiesterase PDE4B limits CAMPassociated PI3K/AKT-dependent apoptosis in diffuse large B-cell lymphoma. Blood 2005, 105:308-316.

24. Kenny HA, Dogan S, Zillhardt M, Zillhardt M, Metra AK, Yamada SD, Krausz T, Lengyel E: Organotypic models of metastasis: A three-dimensional culture mimicking the human peritoneum and omentum for the study of the early steps of ovarian cancer metastasis. Cancer Treat Res 2009, 149:335-351.

25. Tsunoda T, Furusato B, Takashima Y, Ravulapalli S, Dobi A, Srivastava S, McLeod DG, Sesterhenn IA, Ornstein DK, Shirasawa S: The increased expression of periostin during early stages of prostate cancer and advanced stages of cancer stroma. Prostate 2009, 69:1398-1403.

26. Fournier MV, Martin KJ, Kenny PA, Xhaja K, Bosch I, Yaswen P, Bissell MJ: Gene expression signature in organized and growth-arrested mammary acini predicts good outcome in breast cancer. Cancer Res 2006, 66:7095-7102.

27. Martin KJ, Patrick DR, Bissell MJ, Fournier MV: Prognostic breast cancer signature identified from 3D culture model accurately predicts clinical outcome across independent datasets. PLoS One 2008, 3:e2994.

28. Bandrès E, Malumbres R, Cubedo E, Honorato B, Zarate R, Labarga A, Gabisu U, Sola JJ, Garcia-Foncillas J: A gene signature of 8 genes could identify the risk of recurrence and progression in Dukes' B colon cancer patients. Oncol Rep 2007 , 17:1089-1094.

29. Jaffe $A B$, Kaji N, Durgan J, Hall A: Cdc42 controls spindle orientation to position the apical surface during epithelial morphogenesis. J Cell Biol 2008, 183:625-633

30. Martin-Belmonte F, Gassama A, Datta A, Yu W, Rescher U, Gerke V, Mostov K. PTEN-mediated apical segregation of phosphoinositides controls epithelial morphogenesis through Cdc42. Cell 2007, 128:383-397.

31. Kim J, Takeuchi H, Lam ST, Turner RR, Wang HJ, Kuo C, Foshag L, Bilchik AJ Hoon DS: Chemokine receptor CXCR4 expression in colorectal cancer patients increases the risk for recurrence and for poor survival. $J$ Clin Oncol 2005, 23:2744-2753.

32. Zeelenberg IS, Ruuls-Van Stalle L, Roos E: The chemokine receptor CXCR4 is required for outgrowth of colon carcinoma micrometastases. Cancer Res 2003, 63:3833-3839. 
33. Balko JM, Potti A, Saunders C, Stromberg A, Haura EB, Black EP: Gene expression patterns that predict sensitivity to epidermal growth factor receptor tyrosine kinase inhibitors in lung cancer cell lines and human lung tumors. BMC Genomics 2006, 7:289.

34. Park SJ, Ahmad F, Philp A, Baar K, Williams T, Luo H, Ke H, Rehmann H, Taussig R, Brown AL, et al: Resveratrol ameliorates aging-related metabolic phenotypes by inhibiting cAMP phosphodiesterases. Cell 2012, 148:421-433.

35. Kim SW, Rai D, McKeller MR, Aguiar RC: Rational combined targeting of phosphodiesterase 4B and SYK in DLBCL. Blood 2009, 113:6153-6160.

36. Meyers JA, Su DW, Lerner A: Chronic lymphocytic leukemia and B and $T$ cells differ in their response to cyclic nucleotide phosphodiesterase inhibitors. J Immunol 2009, 182:5400-5411.

37. Sengupta R, Sun T, Warrington NM, Rubin JB: Treating brain tumors with PDE4 inhibitors. Trends Pharmacol Sci 2011, 32:337-344.

38. Chen TC, Wadsten P, Su S, Rawlinson N, Hofman FM, Hill CK, Schonthal AH: The type IV phosphodiesterase inhibitor rolipram induces expression of the cell cycle inhibitors p21(Cip1) and p27(Kip1), resulting in growth inhibition, increased differentiation, and subsequent apoptosis of malignant A-172 glioma cells. Cancer Biol Ther 2002, 1:268-276.

39. Murata K, Sudo T, Kameyama M, Fukuoka H, Muka M, Doki Y, Sasaki Y, Ishikawa O, Kimura Y, Imaoka S: Cyclic AMP specific phosphodiesterase activity and colon cancer cell motility. Clin Exp Metastasis 2000, 18:599-604.

40. McEwan DG, Brunton VG, Baillie GS, Leslie NR, Houslay MD, Frame MC: Chemoresistant $\mathrm{KM} 12 \mathrm{C}$ colon cancer cells are addicted to low cyclic AMP levels in a phosphodiesterase 4-regulated compartment via effects on phosphoinositide 3-kinase. Cancer Res 2007, 67:5248-5257.

41. Baba I, Shirasawa S, Iwamoto R, Okumura K, Tsunoda T, Nishioka M, Fukuyama K, Yamamoto K, Mekada E, Sasazuki T: Involvement of deregulated epiregulin expression in tumorigenesis in vivo through activated Ki-Ras signaling pathway in human colon cancer cells. Cancer Res 2000, 60:6886-6889.

42. Tsunoda T, Takashima Y, Yoshida Y, Doi K, Tanaka Y, Fujimoto T, Machida T, Ota T, Koyanagi M, Kuroki M, et al: Oncogenic KRAS Regulates miR-200c and miR-221/222 in a 3D-Specific Manner in Colorectal Cancer Cells. Anticancer Res 2011, 31:2453-2459.

43. Koyanagi M, Nakabayashi K, Fujimoto T, Gu N, Baba I, Takashima Y, Doi K, Harada H, Kato N, Sasazuki T, Shirasawa S: ZFAT expression in B and T lymphocytes and identification of ZFAT-regulated genes. Genomics 2008 91:451-457.

44. Bolstad BM, Irizarry RA, Astrand M, Speed TP: A comparison of normalization methods for high density oligonucleotide array data based on variance and bias. Bioinformatics 2003, 19:185-193.

45. Golub TR, Slonim DK, Tamayo P, Huard C, Gaasenbeek M, Mesirov JP, Coller H, Loh ML, Downing JR, Caligiuri MA, et al: Molecular classification of cancer: class discovery and class prediction by gene expression monitoring. Science 1999, 286:531-537.

46. Barber R, Baillie GS, Bergmann R, Shepherd MC, Sepper R, Houslay MD, Heeke GV: Differential expression of PDE4 CAMP phosphodiesterase isoforms in inflammatory cells of smokers with COPD, smokers without COPD, and nonsmokers. Am J Physiol Lung Cell Mol Physiol 2004, 287:L332-L343.

47. Debnath J, Muthuswamy SK, Brugge JS: Morphogenesis and oncogenesis of MCF-10A mammary epithelial acini grown in three-dimensional basement membrane cultures. Methods 2003, 30:256-268.

48. Yoshida Y, Tsunoda T, Doi K, Tanaka Y, Fujimoto T, Machida T, Ota T, Koyanagi M, Takashima Y, Sasazuki T, et al: KRAS-mediated up-regulation of RRM2 expression is essential for the proliferation of colorectal cancer cell lines. Anticancer Res 2011, 31:2535-2539.

doi:10.1186/1476-4598-11-46

Cite this article as: Tsunoda et al:: Inhibition of Phosphodiesterase-4 (PDE4) activity triggers luminal apoptosis and AKT dephosphorylation in a 3-D colonic-crypt model. Molecular Cancer 2012 11:46.

\section{Submit your next manuscript to BioMed Central and take full advantage of:}

- Convenient online submission

- Thorough peer review

- No space constraints or color figure charges

- Immediate publication on acceptance

- Inclusion in PubMed, CAS, Scopus and Google Scholar

- Research which is freely available for redistribution

Submit your manuscript at www.biomedcentral.com/submit
C Biomed Central 